\title{
Entropic Dynamics: Quantum Mechanics from Entropy and Information Geometry
}

\author{
Ariel Caticha \\ Department of Physics, University at Albany-SUNY, Albany, NY 12222, USA
}

\begin{abstract}
Entropic Dynamics (ED) is a framework in which Quantum Mechanics (QM) is derived as an application of entropic methods of inference. The magnitude of the wave function is manifestly epistemic: its square is a probability distribution. The epistemic nature of the phase of the wave function is also clear: it controls the flow of probability. The dynamics is driven by entropy subject to constraints that capture the relevant physical information. The central concern is to identify those constraints and how they are updated. After reviewing previous work I describe how considerations from information geometry allow us to derive a phase space geometry that combines Riemannian, symplectic, and complex structures. The ED that preserves these structures is QM. The full equivalence between ED and QM is achieved by taking account of how gauge symmetry and charge quantization are intimately related to quantum phases and the single-valuedness of wave functions.
\end{abstract}

\section{Introduction}

Ever since its origin in 1925 the conceptual foundations of Quantum Mechanics (QM) have been a source of controversy. Part of the problem is that quantum mechanics is often conceived as a generalization of classical mechanics with an added quantum indeterminism. The linear Hilbert space structure is given priority while the probabilistic structure is appended almost as an afterthought. The unfortunate consequence is that in the standard approach to QM the dynamical and the probabilistic aspects of quantum theory are not quite compatible with each other. Two separate and irreconcilable modes of wave function evolution are postulated: one is the linear and deterministic Schrödinger evolution and the other is the probabilistic wave function collapse. The problem is that it is not clear why measurement processes should be any different from other regular physical processes. Closely related to these issues is the interpretation of the quantum state itself. Does the wave function represent the actual state of the system (its ontic state) or does it represent our knowledge about the system (an epistemic state):11

\footnotetext{
${ }^{1}$ Excellent reviews with extended references to the literature are given in e.g. [1]-4].
} 
Entropic Dynamics (ED) resolves these problems by placing the probabilistic aspects of QM at the forefront. The Schrödinger equation is derived as an application of entropic methods of inference 2 When constructing models of this kind the first step is to specify the subject matter, the ontology. This step is not trivial: are we talking about particles or about particle detectors? Once the choice is made the inferences are driven by entropy subject to information expressed by constraints. The challenge is to identify the right constraints; it is through them that the "physics" is introduced [9]-13. This view that the wave function $\Psi$ is a fully epistemic concept turns out to be extremely restrictive because it is not sufficient to merely interpret the probability $|\Psi|^{2}$ as a state of knowledge. It is also required that all changes in $\Psi$, which include both the unitary time evolution and the wave function collapse, be obtained as a consequence of entropic and Bayesian updating rules.

The literature on the attempts to reconstruct quantum mechanics is vast (see e.g., 14-20 and references therein) and there are many approaches that are based on information theory (see e.g., $21-(30)$ ). What distinguishes ED is a strict adherence to Bayesian and entropic methods and also a central concern with the nature of time. In ED "entropic" time is a book-keeping device designed to keep track of change. The construction of entropic time involves several ingredients. One must introduce the notion of an 'instant'; one must show that these instants are suitably ordered; and finally one must define a convenient measure of the duration or interval between the successive instants. It turns out that an arrow of time is generated automatically. Entropic time is intrinsically directional.

This paper contains a review of the ED framework [9]-[13] and extends its formalism in three new directions. The first new contribution is a rather straightforward variation on the ED theme. I show that some of the relevant dynamical information - which in the past had been imposed through constraints - can alternatively be supplied through the prior distribution.

The second new contribution builds on and extends recent work in collaboration with N. Carrara. The issue addressed in [13] is whether ED is fully equivalent to $\mathrm{QM}$ or does it merely reproduce a subset of its solutions. This kind of problem was first pointed out long ago by Takabayasi [31] in the context of the hydrodynamical interpretation of QM, and later revived by Wallstrom 32 33 as an objection to Nelson's stochastic mechanics. Wallstrom's objection is that stochastic mechanics leads to phases and wave functions that are either both multi-valued or both single-valued. Both alternatives are unsatisfactory because on one hand QM requires single-valued wave functions, while on the other hand single-valued phases exclude states that are physically relevant (e.g., states with non-zero angular momentum).

The resolution of Wallstrom's objection in the context of ED involves two related ingredients 3 The first recognizes the physical fact that quantum phases

\footnotetext{
${ }^{2}$ The principle of maximum entropy as a method for inference can be traced to the pioneering work of E. T. Jaynes [5]-[7]. For a pedagogical overview of Bayesian and entropic inference and further references see 8 .

${ }^{3} \mathrm{~A}$ hint towards a satisfactory resolution of Wallstrom's objection is found in Takabayasi's
} 
are intimately related to the local electromagnetic gauge symmetry. One cannot properly understand one without taking the other into account. The second ingredient recognizes the experimental fact of charge quantization - that charges occur in multiples of some basic unit. The new and welcome bonus is a deeper understanding of the connection between the probabilistic structure of QM, its linearity, the quantization of charge, and the single-valuedness of wave functions 4

The essence of Entropic Dynamics is the entropic updating of probabilities through information supplied by constraints. The central concern, therefore, is how these constraints are chosen, and in particular, how the constraints themselves are updated. One early insight due to Nelson was that several aspects of quantum behavior could be described as a Brownian motion subject to a conserved energy: stochastic mechanics is a non-dissipative diffusion [37]. Fortunately this was an idea that could be directly imported from Nelson's realistic stochastic mechanics into our epistemic ED. Although in ED energy is a purely epistemic notion, its conservation provides an effective criterion for choosing evolving constraints. This leads to a fully Hamiltonian formalism with its attendant symplectic structure, action principle, Poisson brackets, and so on. Unfortunately this approach, while fully satisfactory in a non-relativistic setting, fails in curved space-times where the concept of a globally conserved energy may not exist.

The third new contribution in this paper is concerned with developing a geometric criterion for updating constraints that does not rely on the notion of a conserved energy. The fact that QM has a rich geometrical structure [38- 43 including a deep connection to information geometry [21] has been explored by many authors. There is, for example, the application to quantum statistical inference developed by Brody and Hughston [44, and the operational framework for the analysis of measurements described in the work of Mehrafarin 24] and Goyal [26]. The work of Reginatto and Hall [29] 30] is particularly relevant to us. They impose a symplectic structure in the phase space $\{\rho, \Phi\}$ of probability distributions $\rho$ and their conjugate momenta $\Phi$. To motivate the choice of Hamiltonian flow they extend the natural information metric over the ensemble space $\{\rho\}$ to the whole phase space $\{\rho, \Phi\}$ in a way that maintains the symplectic structure.

The approach we pursue here differs by virtue of being based on a different epistemic goal - to identify constraints for entropic inference. More specifically we derive the symplectic structure rather than impose it as a starting point. In ED the degrees of freedom are the probability densities $\rho(x)$ and the phase fields $\Phi(x)$. The latter represent the constraints that control the flow of probabilities. Our goal too is to extend the information geometry from the "ensemble" space $\{\rho\}$ to the full "ensemble-phase space" $\{\rho, \Phi\}$, which we abbreviate as "e-phase"

later work which incorporates spin into his hydrodynamical approach 34]. Although here we focus on non-spinning particles our choice of constraints can be generalized to particles with spin $1 / 2$ - a project to be addressed in a future publication.

${ }^{4}$ The issue of single-valuedness has been discussed from the different perspective of group representations by Pauli [35] 36]. 
space. To do this we impose a symmetry that is natural in a probabilistic setting: we extend the well-known spherically symmetric information geometry from the ensemble space $\{\rho\}$ to the full e-phase space $\{\rho, \Phi\}$. Then we show that the extended metric describes quantum geometry, that is, it includes the Riemannian, the complex, and the symplectic structures that we recognize as quantum geometry.

The desired geometric criterion for updating constraints is a dynamics that preserves these structures. Therefore ED is a Hamiltonian dynamics. The most natural Hamiltonian is simply constructed from the extended metric by recognizing the privileged ontic role of position. The resulting dynamics is described by the Schrödinger equation.

This paper focuses on the derivation of the Schrödinger equation but the ED approach has been applied to a variety of other topics including the quantum measurement problem [45] 46]; momentum and uncertainty relations [47; the Bohmian limit [48] 49] and the classical limit [50]; extensions to curved spaces 51] and to relativistic fields 52 53] are also available.

\section{The statistical model}

We consider $N$ particles living in a flat Euclidean space $\mathbf{X}$ with metric $\delta_{a b}$. The first assumption is that the particles have definite positions $x_{n}^{a}$, collectively denoted by $x$, and it is their unknown values that we wish to infer. (The index $n=1 \ldots N$ labels the particles, and $a=1,2,3$ the three spatial coordinates.) The configuration space for $N$ particles is $\mathbf{X}_{N}=\mathbf{X} \times \ldots \times \mathbf{X}$.

Since the positions are unknown the main target of our attention is the probability distribution $\rho(x)$. Incidentally, this already addresses that old question of determinism vs. indeterminism that has troubled quantum mechanics from the outset. Inference techniques are designed to cope with insufficient information. In an inference approach to quantum theory one starts by accepting uncertainty, probability, and indeterminism as the expected and inevitable norm that requires no explanation; it is the certainty and determinism of classical mechanics that demand explanations (see e.g., [50]).

The assumption that the particles have definite positions that happen to be unknown is a major departure from the standard Copenhagen interpretation according to which observable quantities do not in general have definite values. In the standard approach observables can attain definite values but only as the result of a measurement. In contrast, positions in ED play a very special role: they define the ontic state of the system. For example, in the double slit experiment, we might not know which slit the particle goes through, but the particle definitely goes through one slit or the other. The wave function, on the other hand, is a purely epistemic notion; it defines our epistemic state about the system. All other quantities, such as energy or momentum, are epistemic in that they reflect properties of the wave function, not properties of the particles [45- 477. These values are not quite "created" by the measurement, but rather inferred from them. Such quantities may, therefore, be more aptly referred to 
as "inferables" rather than "observables" 5

Once the microstates $x \in \mathbf{X}_{N}$ are identified we proceed to the dynamics. The goal is to find the probability density $P\left(x^{\prime} \mid x\right)$ of a step from an initial position $x \in \mathbf{X}_{N}$ to a new neighboring $x^{\prime} \in \mathbf{X}_{N}$ by maximizing the entropy,

$$
\mathcal{S}[P, Q]=-\int d x^{\prime} P\left(x^{\prime} \mid x\right) \log \frac{P\left(x^{\prime} \mid x\right)}{Q\left(x^{\prime} \mid x\right)},
$$

relative to a prior $Q\left(x^{\prime} \mid x\right)$, and subject to the appropriate constraints specified below. (For notational simplicity in multidimensional integrals such as (1) we will write $d x^{\prime}$ instead of $d^{n} x^{\prime}$.)

The prior- In ED one does not explain why motion happens - this is a "mechanics without a mechanism." Instead our task is to produce an estimate of what kind of motion one might reasonably expect. The main dynamical assumption is that the particles follow trajectories that are continuous. This introduces an enormous simplification because it implies that motion can be analyzed as the accumulation of many infinitesimally short steps and, therefore, our first task is to find the transition probability $P\left(x^{\prime} \mid x\right)$ for an infinitesimally short step.

The prior $Q\left(x^{\prime} \mid x\right)$ in eq.(11) expresses our state of knowledge before any information about the details of a particular motion are taken into account. Accordingly, we adopt a prior that incorporates the information that the particles' steps are infinitesimally short, but is otherwise maximally uninformative. We want a prior that reflects translational and rotational invariance, and is ignorant about any correlations. Such a prior can itself be derived from the principle of maximum entropy. Indeed, maximize

$$
S[Q]=-\int d x^{\prime} Q(\Delta x) \log \frac{Q(\Delta x)}{\mu(\Delta x)},
$$

where $\Delta x_{n}^{a}=x_{n}^{\prime a}-x_{n}^{a}$, relative to the uniform measure $\mu(\Delta x)$, subject to normalization, and $N$ independent constraints that enforce short steps and rotational invariance,

$$
\left\langle\delta_{a b} \Delta x_{n}^{a} \Delta x_{n}^{b}\right\rangle=\kappa_{n}, \quad(n=1 \ldots N),
$$

where $\kappa_{n}$ are small constants. The result is a product of Gaussians,

$$
Q\left(x^{\prime} \mid x\right) \propto \exp -\frac{1}{2} \sum_{n} \alpha_{n} \delta_{a b} \Delta x_{n}^{a} \Delta x_{n}^{b},
$$

where the $\alpha_{n}$ are Lagrange multipliers that will eventually be taken to infinity in order to enforce infinitesimally short steps. For now we just anticipate that the $\alpha_{n}$ are constants that are independent of $x$ but may depend on the index $n$ in order to describe non-identical particles. Next we specify the constraints.

\footnotetext{
${ }^{5}$ I thank Kevin Vanslette for suggesting this terminology.
} 
The drift potential constraint- The physical information that the motion of the particles can be both directional and highly correlated - including such effects as entanglement - is introduced through a single constraint involving a "drift" potential $\phi(x)=\phi\left(x_{1} \ldots x_{N}\right)$ that is a function in configuration space, $x \in \mathbf{X}_{N}$. We impose that the displacements $\Delta x_{n}^{a}$ are such that the expected change of the drift potential $\langle\Delta \phi\rangle$ is constrained to be

$$
\langle\Delta \phi\rangle=\sum_{n=1}^{N}\left\langle\Delta x_{n}^{a}\right\rangle \frac{\partial \phi}{\partial x_{n}^{a}}=\kappa^{\prime},
$$

where $\kappa^{\prime}$ is another small but for now unspecified position-independent constant.

The introduction of the drift potential $\phi(x)$ will not be justified at this point but, given its importance, a couple of brief comments may be of value. First, we note that one can make progress by identifying the relevant constraints even when their physical origin remains unexplained. This situation is not unlike classical mechanics where identifying the forces can be very useful even in those situations where their microscopic origin is not yet fully understood. Second, it is in fact possible to provide a microscopic interpretation of $\phi(x)$ in terms of the entropy of some other microscopic variables that live at some deeper "subquantum" level [9]. Whether postulating such variables leads to further physical insights is an interesting topic for future research.

The gauge constraints- The minimal assumptions described in the previous paragraphs already lead to an interesting ED but we can construct richer forms of dynamics that describe electromagnetic interactions by imposing additional constraints.

We assume that the motion of each particle $n$ is affected by an additional field $\chi\left(x_{n}\right)\left(x_{n} \in \mathbf{X}\right.$ is a point in physical 3D space) with the topological properties of an angle: $\chi\left(x_{n}\right)$ and $\chi\left(x_{n}\right)+2 \pi$ describe the same angle. We further assume that these angles can be redefined by different amounts $\gamma(x)$ at different places, that is, the origin from which these angles are measured can be set independently at each $x$. This is a local gauge symmetry and it immediately raises the question of how can one compare angles at different locations. The answer is well known: introduce a connection field, a vector potential $A_{a}(x)$ that defines which angle at $x+\Delta x$ is the "same" as the angle at $x$. This is implemented by imposing that as $\chi \rightarrow \chi+\gamma$ then the connection transforms as $A_{a} \rightarrow A_{a}+\partial_{a} \gamma$ so that the corrected derivative $\partial_{a} \chi-A_{a}$ remains invariant 6 To derive an ED that incorporates gauge invariant interactions for each particle $n$ we impose a constraint that involves these corrected derivatives,

$$
\left\langle\Delta x_{n}^{a}\right\rangle\left[\partial_{n a} \chi\left(x_{n}\right)-A_{a}\left(x_{n}\right)\right]=\kappa_{n}^{\prime \prime}, \quad(n=1 \ldots N),
$$

where $\partial_{n a}=\partial / \partial x_{n}^{a}$. The quantities $\kappa_{n}^{\prime \prime}$ can be specified directly, but as is often the case in entropic inference, it is much more convenient to specify the

\footnotetext{
${ }^{6}$ Note that since $\chi$ is dimensionless the vector potential $A_{a}$ has units of inverse length. This defines units of electric charge that differ from those conventionally adopted in electromagnetism.
} 
constraints indirectly through the corresponding Lagrange multipliers $\beta_{n}$ which, as we shall later see, will turn out to be the electric charges.

The transition probability - The distribution $P\left(x^{\prime} \mid x\right)$ that maximizes the entropy $\mathcal{S}[P, Q]$ in (1) relative to (4) and subject to (5), (6), and normalization is

$$
P\left(x^{\prime} \mid x\right) \propto \exp \sum_{n}\left[-\frac{\alpha_{n}}{2} \delta_{a b} \Delta x_{n}^{a} \Delta x_{n}^{b}+\alpha^{\prime} \frac{\partial \phi}{\partial x_{n}^{a}} \Delta x_{n}^{a}+\beta_{n}\left(\partial_{n a} \chi_{n}-A_{a}\left(x_{n}\right)\right) \Delta x_{n}^{a}\right]
$$

where $\alpha^{\prime}$ and $\beta_{n}$ are Lagrange multipliers. It is convenient to rewrite $P\left(x^{\prime} \mid x\right)$ as

$$
P\left(x^{\prime} \mid x\right)=\frac{1}{Z} \exp \left[-\frac{1}{2} \sum_{n} \alpha_{n} \delta_{a b}\left(\Delta x_{n}^{a}-\left\langle\Delta x_{n}^{a}\right\rangle\right)\left(\Delta x_{n}^{b}-\left\langle\Delta x_{n}^{b}\right\rangle\right)\right]
$$

where $Z$ is a normalization constant. Thus, a generic displacement $\Delta x_{n}^{a}=$ $x_{n}^{\prime a}-x_{n}^{a}$ can be expressed as an expected drift plus a fluctuation,

$$
\Delta x_{n}^{a}=\left\langle\Delta x_{n}^{a}\right\rangle+\Delta w_{n}^{a},
$$

where

$$
\begin{gathered}
\left\langle\Delta x_{n}^{a}\right\rangle=\frac{1}{\alpha_{n}} \delta^{a b}\left[\alpha^{\prime} \partial_{n b} \phi+\beta_{n} \partial_{n b} \chi\left(x_{n}\right)-\beta_{n} A_{b}\left(x_{n}\right)\right] \\
\left\langle\Delta w_{n}^{a}\right\rangle=0 \quad \text { and }\left\langle\Delta w_{n}^{a} \Delta w_{n}^{b}\right\rangle=\frac{1}{\alpha_{n}} \delta^{a b}
\end{gathered}
$$

We see that for very short steps, as $\alpha_{n} \rightarrow \infty$, the dynamics is dominated by fluctuations $\Delta w_{n}^{a}$ which are of order $O\left(\alpha_{n}^{-1 / 2}\right)$, while the drift $\left\langle\Delta x_{n}^{a}\right\rangle$ is much smaller, only of order $O\left(\alpha_{n}^{-1}\right)$. Thus, just as in Brownian motion, the trajectory is continuous but not differentiable. In ED particles have definite positions but their velocities are completely undefined. The directionality of the motion and the correlations among the particles are introduced by a systematic drift determined by the drift potential $\phi$ and the gauge fields $\chi$ and $A_{a}$, while the particle fluctuations remain isotropic and uncorrelated. We can also see that the effect of $\alpha^{\prime}$ is to enhance or suppress the magnitude of the drift relative to the fluctuations. The large $\alpha^{\prime}$ limit turns out to be the Bohmian limit of ED (see e.g., 48 49]). However, since the drift potential is at this point unspecified, we can without loss of generality absorb $\alpha^{\prime}$ into $\phi, \alpha^{\prime} \phi \rightarrow \phi$, which amounts to setting $\alpha^{\prime}=1$.

\section{Entropic time}

Having obtained a prediction, given by eq.(8), for what motion to expect in one infinitesimally short step we now consider motion over finite distances. This requires the introduction of a parameter $t$, to be called time, as a book-keeping tool to keep track of the accumulation of short steps. The construction of time involves three ingredients. First, we must identify something that one might call an "instant"; second, it must be shown that these instants are ordered; and 
finally, one must introduce a measure of separation between these successive instants - one must define "duration." Since the foundation for any theory of time is the theory of change - that is, the underlying dynamics - the notion of time constructed below will reflect the inferential nature of entropic dynamics. Such a construction we will call entropic time [9].

Time as an ordered sequence of instants- Entropic dynamics is given by a succession of short steps described by $P\left(x^{\prime} \mid x\right)$, eq. (8). Consider, for example, the $i$ th step which takes the system from $x=x_{i-1}$ to $x^{\prime}=x_{i}$. Integrating the joint probability, $P\left(x_{i}, x_{i-1}\right)$, over $x_{i-1}$ gives

$$
P\left(x_{i}\right)=\int d x_{i-1} P\left(x_{i}, x_{i-1}\right)=\int d x_{i-1} P\left(x_{i} \mid x_{i-1}\right) P\left(x_{i-1}\right) .
$$

This equation follows directly from the laws of probability, it involves no physical assumptions and, therefore, it is sort of empty. To make it useful something else must be added. Suppose we interpret $P\left(x_{i-1}\right)$ as the probability of different values of $x_{i-1}$ at one "instant" labelled $t$, then we can interpret $P\left(x_{i}\right)$ as the probability of values of $x_{i}$ at the next "instant" which we will label $t^{\prime}$. Writing $P\left(x_{i-1}\right)=\rho_{t}(x)$ and $P\left(x_{i}\right)=\rho_{t^{\prime}}\left(x^{\prime}\right)$ we have

$$
\rho_{t^{\prime}}\left(x^{\prime}\right)=\int d x P\left(x^{\prime} \mid x\right) \rho_{t}(x)
$$

Nothing in the laws of probability leading to eq. (12) forces the interpretation (13) on us - this is the additional ingredient that allows us to construct time and dynamics in our model. Equation (13) defines the notion of "instant": If the distribution $\rho_{t}(x)$ refers to one instant $t$, then the distribution $\rho_{t^{\prime}}\left(x^{\prime}\right)$ generated by $P\left(x^{\prime} \mid x\right)$ defines what we mean by the "next" instant $t^{\prime}$. The dynamics is defined by iterating this process. Entropic time is constructed instant by instant: $\rho_{t^{\prime}}$ is constructed from $\rho_{t}, \rho_{t^{\prime \prime}}$ is constructed from $\rho_{t^{\prime}}$, and so on.

The construction is intimately related to information and inference. An 'instant' is an informational state that is complete in the sense that it is specified by the information - codified into the distributions $\rho_{t}(x)$ and $P\left(x^{\prime} \mid x\right)$ - that is sufficient for predicting the next instant. Thus, the present is defined such that, given the present, the future is independent of the past.

In the ED framework the notions of instant and of simultaneity are intimately related to the distribution $\rho_{t}(x)$. It is instructive to discuss this further. When we consider a single particle at a position $\vec{x}=\left(x^{1}, x^{2}, x^{3}\right)$ in $3 \mathrm{D}$ space it is implicit in the notation that the three coordinates $x^{1}, x^{2}$, and $x^{3}$ occur simultaneously — no surprises here. Things get a bit more interesting when we describe a system of $N$ particles by a single point $x=\left(\vec{x}_{1}, \vec{x}_{2}, \ldots \vec{x}_{N}\right)$ in $3 N$-dimensional configuration space. Here it is also implicitly assumed that all the $3 N$ coordinate values refer to the same instant; we take them to be simultaneous. There is an implicit assumption linking the very idea of a point in configuration space with that of simultaneity. Whether we talk about one particle or about $N$ particles, a distribution such as $\rho_{t}(x)$ describes our uncertainty 
about the possible configurations $x$ of the system at a given instant. Thus, in $\mathrm{ED}$, a probability distribution $\rho_{t}(x)$ provides a criterion of simultaneity.

In a relativistic theory there is a greater freedom in the choice of instants and this translates into a greater flexibility with the notion of simultaneity. Conversely, as we have shown elsewhere, the requirement that these different notions of simultaneity be consistent with each other places strict constraints on the allowed forms of relativistic ED [53].

It is common to use equations such as (13) to define a special kind of dynamics, called Markovian, that unfolds in a time defined by some external clocks. In such a Markovian dynamics the specification of the state at one instant is sufficient to determine its evolution into the future. It is important to emphasize that although the ED eq. (13) is formally identical to the Chapman-Kolmogorov equation we are not making a Markovian assumption. We do not use (13) to define a (Markovian) dynamics in a pre-existing background time because in ED there are no external clocks. The system provides its own clock and (13) is used both to define the dynamics and to construct time itself. In this respect, entropic time bears some resemblance with the relational notion of time advocated by J. Barbour in the context of classical physics (see e.g. [54]).

The arrow of entropic time-- The notion of time constructed according to eq.(13) is intrinsically directional. There is an absolute sense in which $\rho_{t}(x)$ is prior and $\rho_{t^{\prime}}\left(x^{\prime}\right)$ is posterior. If we wanted to construct a time-reversed evolution we would write

$$
\rho_{t}(x)=\int d x^{\prime} P\left(x \mid x^{\prime}\right) \rho_{t^{\prime}}\left(x^{\prime}\right),
$$

where according to the rules of probability theory $P\left(x \mid x^{\prime}\right)$ is related to $P\left(x^{\prime} \mid x\right)$ in eq.(8) by Bayes' theorem,

$$
P\left(x \mid x^{\prime}\right)=\frac{\rho_{t}(x)}{\rho_{t^{\prime}}\left(x^{\prime}\right)} P\left(x^{\prime} \mid x\right) .
$$

Note, however, that this is not a mere exchange of primed and unprimed quantities. The distribution $P\left(x^{\prime} \mid x\right)$, eq.(8) , is a Gaussian derived from the maximum entropy method. In contrast, the time-reversed $P\left(x \mid x^{\prime}\right)$ is given by Bayes' theorem, eq.15), and is not in general Gaussian. In (15) we see that the asymmetry between the inferential past and the inferential future arises from the asymmetry between priors and posteriors.

The puzzle of the arrow of time (see e.g. [55] [56]) has been how to explain the asymmetric arrow from underlying symmetric laws. The solution offered by $\mathrm{ED}$ is that there are no underlying laws whether symmetric or not. The time asymmetry is the inevitable consequence of entropic inference. From the point of view of ED the challenge is not to explain the arrow of time but the reverse: how to explain the emergence of symmetric laws within an entropic framework that is intrinsically asymmetric. As we shall see below some laws of physics derived from ED, such as the Schrödinger equation, are indeed time-reversible even though entropic time itself is strongly directional. 
Duration: a convenient time scale- To complete the construction of entropic time we need to specify the interval $\Delta t$ between successive instants. The basic criterion is convenience: duration is defined so that motion looks simple. We saw in eqs.(10) and (11) that for short steps (large $\alpha_{n}$ ) the motion is largely dominated by fluctuations. Therefore specifying $\Delta t$ amounts to specifying the multipliers $\alpha_{n}$ in terms of $\Delta t$.

The description of motion is simplest when it reflects the symmetry of translations in space and time. In a flat spacetime this leads us to an entropic time that resembles Newtonian time in that it flows "equably everywhere and everywhen." Thus, we choose $\alpha_{n}$ to be independent of $x$ and $t$, and we choose $\Delta t$ so that $\alpha_{n} \propto 1 / \Delta t$. Furthermore, it is convenient to express the proportionality constants in terms of some particle-specific constants $m_{n}$ and an overall constant $\hbar$ that fixes the units of the $m_{n}$ s relative to the units of time. The result is

$$
\alpha_{n}=\frac{m_{n}}{\hbar} \frac{1}{\Delta t} .
$$

As we shall see, the constants $m_{n}$ will eventually be identified with the particle masses while the constant $\hbar$ will be identified as Planck's constant.

\section{The information metric of configuration space}

Before we proceed to study the dynamics defined eq.(8) it is useful to consider the geometry of the $N$-particle configuration space, $\mathbf{X}_{N}$. Since the physical single particle space $\mathbf{X}$ is described by the Euclidean metric $\delta_{a b}$ we can expect that the $N$-particle configuration space, $\mathbf{X}_{N}=\mathbf{X} \times \ldots \times \mathbf{X}$, will also be flat, but for non-identical particles a question remains about the relative weights associated to each $\mathbf{X}$ factor. Information geometry provides the answer.

To each point $x \in \mathbf{X}_{N}$ there corresponds a probability distribution $P\left(x^{\prime} \mid x\right)$. This means that to the configuration space $\mathbf{X}_{N}$ we can associate a statistical manifold and its geometry is uniquely determined (up to an overall scale factor) by the information metric,

$$
\gamma_{A B}=C \int d x^{\prime} P\left(x^{\prime} \mid x\right) \frac{\partial \log P\left(x^{\prime} \mid x\right)}{\partial x^{A}} \frac{\partial \log P\left(x^{\prime} \mid x\right)}{\partial x^{B}} .
$$

Here the upper case indices label both the particle and its coordinate, $x^{A}=x_{n}^{a}$, and $C$ is an arbitrary positive constant (see e.g., [57 [8]). Substituting eqs. (8) and (16) into (17) in the limit of short steps $\left(\alpha_{n} \rightarrow \infty\right)$ yields

$$
\gamma_{A B}=\frac{C m_{n}}{\hbar \Delta t} \delta_{n n^{\prime}} \delta_{a b}=\frac{C m_{n}}{\hbar \Delta t} \delta_{A B} .
$$

The divergence as $\Delta t \rightarrow 0$ arises because the information metric measures statistical distinguishability. As $\Delta t \rightarrow 0$ the distributions $P\left(x^{\prime} \mid x\right)$ and $P\left(x^{\prime} \mid x+\Delta x\right)$ become more sharply peaked and increasingly easier to distinguish. Therefore, $\gamma_{A B} \rightarrow \infty$. To define a geometry that remains useful even for arbitrarily small 
$\Delta t$ we choose $C \propto \Delta t$. In fact, since $\gamma_{A B}$ will always appear in the combination $\gamma_{A B} \Delta t / C$, it is best to absorb these constants into a "mass" tensor,

$$
m_{A B}=\frac{\hbar \Delta t}{C} \gamma_{A B}=m_{n} \delta_{A B} .
$$

The inverse mass tensor will also turn out to be useful,

$$
m^{A B}=\frac{C}{\hbar \Delta t} \gamma^{A B}=\frac{1}{m_{n}} \delta^{A B} .
$$

Thus, up to overall constants the mass tensor is the metric of configuration space. Ever since the work of Heinrich Hertz in 1894 [58] it has been standard practice to describe the motion of systems with many particles as the motion of a single point in an abstract space - the configuration space. Choosing the geometry of this configuration space based on an examination of the kinetic energy of the system has so far been regarded as a matter of convenience. In ED there is no choice: up to a global scale factor the metric follows uniquely from information geometry.

To summarize our results so far: with the constants $\alpha_{n}$ chosen according to (16), the dynamics given by $P\left(x^{\prime} \mid x\right)$ in (8) is a standard Wiener process. A generic displacement is written as a drift plus a fluctuation,

$$
\Delta x^{A}=b^{A} \Delta t+\Delta w^{A} .
$$

From eq.(10) the drift velocity is

$$
b^{A}=\frac{\left\langle\Delta x^{A}\right\rangle}{\Delta t}=m^{A B}\left[\partial_{B} \hbar(\phi+\bar{\chi})-\bar{A}_{B}\right],
$$

where we have introduced the configuration space quantities,

$$
\bar{\chi}(x)=\sum_{n} \beta_{n} \chi\left(x_{n}\right) \quad \text { and } \quad \bar{A}_{A}(x)=\hbar \beta_{n} A_{a}\left(x_{n}\right),
$$

and the fluctuation $\Delta w^{A}$ is given by

$$
\left\langle\Delta w^{A}\right\rangle=0 \quad \text { and } \quad\left\langle\Delta w^{A} \Delta w^{B}\right\rangle=\hbar m^{A B} \Delta t .
$$

I finish this section with two remarks. The first is on the nature of clocks: In Newtonian mechanics time is defined to simplify the motion of free particles. The prototype of a clock is a free particle which moves equal distances in equal times. In ED time is also defined to simplify the dynamics of free particles (for sufficiently short times all particles are free) and the prototype of a clock is a free particle too: as we see in (24) a free particle's variance increases by equal amounts in equal times.

The second remark is on the nature of mass. In standard quantum mechanics, "what is mass?" and "why quantum fluctuations?" are two independent mysteries. In ED the mystery is somewhat alleviated: mass and fluctuations are two sides of the same coin. Mass is an inverse measure of fluctuations. 


\section{Diffusive dynamics}

The dynamics of $\rho(x)$, given by the integral equation (13), is more conveniently re-written in a differential form known as the Fokker-Planck (FP) equation,

$$
\partial_{t} \rho=-\partial_{A}\left(b^{A} \rho\right)+\frac{1}{2} \hbar m^{A B} \partial_{A} \partial_{B} \rho
$$

(For the algebraic details see e.g., [8].) The FP equation can also be written as a continuity equation,

$$
\partial_{t} \rho=-\partial_{A}\left(\rho v^{A}\right) \text {. }
$$

The product $\rho v^{A}$ in (26) represents the probability current, and $v^{A}$ is interpreted as the velocity of the probability flow - it is called the current velocity. From (25) the current velocity in (26) is the sum of two separate contributions,

$$
v^{A}=b^{A}+u^{A} .
$$

where $u^{A}$ is the osmotic velocity,

$$
u^{A}=-\hbar m^{A B} \partial_{B} \log \rho^{1 / 2} .
$$

It represents diffusion, the tendency for probability to flow down the density gradient. Since both $b^{A}$ and $u^{A}$ involve gradients the current velocity can be written in gauge invariant form,

$$
v^{A}=m^{A B}\left(\partial_{B} \Phi-\bar{A}_{B}\right) \quad \text { where } \Phi=\hbar\left(\phi+\bar{\chi}-\log \rho^{1 / 2}\right) .
$$

The field $\Phi$, which we will call the "phase", plays a central role in what follows. The FP equation (26) can be conveniently rewritten in yet another equivalent but very suggestive form involving functional derivatives. For some suitably chosen functional $\tilde{H}[\rho, \Phi]$ we have

$$
\partial_{t} \rho(x)=-\partial_{A}\left[\rho m^{A B}\left(\partial_{B} \Phi-\bar{A}_{B}\right)\right]=\frac{\delta \tilde{H}}{\delta \Phi(x)} .
$$

It is easy to check that the appropriate functional $\tilde{H}$ is

$$
\tilde{H}[\rho, \Phi]=\int d x \frac{1}{2} \rho m^{A B}\left(\partial_{A} \Phi-\bar{A}_{A}\right)\left(\partial_{B} \Phi-\bar{A}_{B}\right)+F[\rho],
$$

where the unspecified functional $F[\rho]$ is an integration constant.

Equation (30) describes a standard diffusion involving a single dynamical field $\rho(x)$ that evolves in response to the non-dynamical fields $\phi, \bar{\chi}$, and $\bar{A}$. In contrast, a quantum dynamics consists in the coupled evolution of two dynamical fields: the density $\rho(x)$ and the phase of the wave function. This second field can be naturally introduced into ED by allowing the field $\Phi$ in (29) to become dynamical: the phase $\Phi$ guides the evolution of $\rho$, and in return, the evolving $\rho$ reacts back and induces a change in $\Phi$. This amounts to an ED in which the constraint (5) is continuously updated at each instant in time. To find the appropriate updating criterion, which gives the dynamics in e-phase space we appeal once again to information geometry. 


\section{The geometry of e-phase space}

It may be convenient to recall one particular derivation of the information metric. In the discrete case the statistical manifold is the simplex $\mathcal{S}_{\nu-1}=\{\rho=$ $\left.\left(\rho^{1} \ldots \rho^{\nu}\right): \sum_{i}^{\nu} \rho^{i}=1\right\}$. As is common in the context of probability and inference we appeal to symmetry. Changing to new coordinates $\xi^{i}=\left(\rho^{i}\right)^{1 / 2}$ the equation for the simplex $\mathcal{S}_{\nu-1}$ - the normalization condition - reads $\sum_{i}^{\nu}\left(\xi^{i}\right)^{2}=1$ which suggests the equation of a sphere. We take this hint seriously and declare that the symplex is an $(\nu-1)$-sphere embedded in an $\nu$-dimensional spherically symmetric space 7 The metric of a generic spherically symmetric space takes the form

$$
d \ell^{2}=(a(|\rho|)-b(|\rho|))\left(\sum_{i}^{\nu} \xi^{i} d \xi^{i}\right)^{2}+|\rho| b(|\rho|) \sum_{i}^{\nu}\left(d \xi^{i}\right)^{2},
$$

where $a(|\rho|)$ and $b(|\rho|)$ are two arbitrary smooth and positive functions of $|\rho|=$ $\sum_{i}^{\nu} \rho^{i}$. Changing back to the original $\rho^{i}$ coordinates and setting $|\rho|=1$ and $\sum_{i}^{\nu} d \rho^{i}=0$ gives the information metric up to an overall scale,

$$
d \ell^{2}=b(1) \sum_{i}^{\nu} \frac{1}{\rho^{i}}\left(d \rho^{i}\right)^{2} .
$$

To extend the information metric from the symplex $\mathcal{S}_{\nu-1}$ to the $2 \nu$-dimensional e-phase space $\left(\rho^{i}, \Phi^{i}\right)$ we impose two conditions: (A) that the extended metric be compatible with the information metric on the symplex $\mathcal{S}_{\nu-1}$, and (B) that $\Phi$ inherits from the gauge potential $\chi$ the topological structure of an angle. The simplest way to implement (A) is to follow as closely as possible the derivation that led to (33). Condition (B) suggests introducing coordinates,

$$
\xi^{i}=\left(\rho^{i}\right)^{1 / 2} \cos \Phi^{i} / \hbar \text { and } \eta^{i}=\left(\rho^{i}\right)^{1 / 2} \sin \Phi^{i} / \hbar .
$$

Then the normalization condition reads

$$
|\rho|=\sum_{i}^{\nu} \rho^{i}=\sum_{i}^{\nu}\left[\left(\xi^{i}\right)^{2}+\left(\eta^{i}\right)^{2}\right]=1
$$

which suggests the equation of a sphere in $2 \nu$ dimensions and, once again, we take the spherical symmetry seriously. The most general metric in the space $\{\rho, \Phi\}$ that is invariant under rotations is

$$
d \ell^{2}=(a(|\rho|)-b(|\rho|))\left[\sum_{i}^{\nu}\left(\xi^{i} d \xi^{i}+\eta^{i} d \eta^{i}\right)\right]^{2}+|\rho| b(|\rho|) \sum_{i}^{\nu}\left[\left(d \xi^{i}\right)^{2}+\left(d \eta^{i}\right)^{2}\right] .
$$

Transforming back to the coordinates $\left(\rho^{i}, \Phi^{i}\right)$, setting $|\rho|=1$ and $\sum_{i}^{\nu} d \rho^{i}=0$, and dropping an unimportant proportionality constant, leads to

$$
d \ell^{2}=\sum_{i}^{\nu}\left[\frac{\hbar}{2 \rho^{i}}\left(d \rho^{i}\right)^{2}+\frac{2}{\hbar} \rho^{i}\left(d \Phi^{i}\right)^{2}\right] .
$$

Finally, generalizing to the continuous e-phase space gives the desired result,

$$
\delta \ell^{2}=\int d x\left[\frac{\hbar}{2 \rho} \delta \rho^{2}+\frac{2}{\hbar} \rho \delta \Phi^{2}\right] .
$$

\footnotetext{
${ }^{7}$ One might be concerned that this particular choice of symmetry is an ad hoc assumption but the result is very robust. Exactly the same metric is obtained by several different criteria [59] 8 .
} 
Index notation- To deal with tensors in e-phase space we introduce a convenient index notation. A point $X$ will be labelled by its coordinates $X^{\alpha x}$, where $\alpha$ takes one of two values, $\rho$ or $\Phi$. Thus,

$$
X^{\alpha x}=\left(X^{\rho x}, X^{\Phi x}\right)=(\rho(x), \Phi(x)) .
$$

Repeated indices will as usual imply sums and integrals. For example, eq.(38) is written as

$$
\delta \ell^{2}=\int d x d x^{\prime} G_{\alpha \alpha^{\prime}}\left(x, x^{\prime}\right) \delta X^{\alpha x} \delta X^{\alpha^{\prime} x^{\prime}}=G_{\alpha x, \beta x^{\prime}} \delta X^{\alpha x} \delta X^{\beta x^{\prime}}
$$

where the metric tensor $G$ has components $G_{\alpha x, \beta x^{\prime}}$,

$$
G_{\alpha x, \beta x^{\prime}}=G_{\alpha \beta}\left(x, x^{\prime}\right)=\left[\begin{array}{cc}
\hbar / 2 \rho(x) & 0 \\
0 & 2 \rho(x) / \hbar
\end{array}\right] \delta\left(x, x^{\prime}\right) .
$$

The scalar product of two vectors $V^{\alpha x}$ and $U^{\beta x^{\prime}}$ is

$$
G[V, U]=G_{\alpha x, \beta x^{\prime}} V^{\alpha x} U^{\beta x^{\prime}} .
$$

Complex and Symplectic structures- A linear transformation $V \rightarrow J V=$ $V_{J}$ or, more explicitly,

$$
V_{J}^{\alpha x}=J_{\beta x^{\prime}}^{\alpha x} V^{\beta x^{\prime}}=\int d x^{\prime} J_{\beta}^{\alpha}\left(x, x^{\prime}\right) V^{\beta x^{\prime}},
$$

is said to be a symmetry of the metric $G$, and we say that $G$ is $J$-invariant, when

$$
G\left[V_{J}, U_{J}\right]=G[V, U]
$$

for all vectors $V$ and $U$. Equivalently, $G$ is $J$-invariant when

$$
G_{\alpha x, \beta x^{\prime}} J_{\gamma x^{\prime \prime}}^{\alpha x} J_{\delta x^{\prime \prime \prime}}^{\beta x^{\prime}}=G_{\gamma x^{\prime}, \delta x^{\prime \prime \prime}} .
$$

It turns out that, beyond spherical symmetry, the metric (41) has another crucial symmetry. It is invariant under the transformation

$$
J_{\beta x^{\prime}}^{\alpha x}=J_{\beta}^{\alpha}\left(x, x^{\prime}\right)=\left[\begin{array}{cc}
0 & 2 \rho(x) / \hbar \\
-\hbar / 2 \rho(x) & 0
\end{array}\right] \delta\left(x, x^{\prime}\right) .
$$

The proof is straightforward: just substitute (46) to verify it satisfies (45).

The importance of $J$ invariance is twofold. First, applying $J$ twice gives $J^{2}=-\mathbf{1}$, or

$$
J^{\alpha x}{ }_{\beta x^{\prime}} J^{\beta x^{\prime}}{ }_{\gamma x^{\prime \prime}}=-\delta^{\alpha x} \gamma x^{\prime \prime}=-\delta_{\gamma}^{\alpha} \delta\left(x, x^{\prime \prime}\right) .
$$

Therefore the metric (38) automatically endows e-phase space with a complex structure $J^{\alpha x} \beta_{x^{\prime}}$. This is the reason why complex numbers play such a central role of quantum mechanics. Second, we can use the metric tensor to lower the first index in $J$,

$$
G_{\alpha x, \beta x^{\prime}} J^{\beta x^{\prime}}{ }_{\gamma x^{\prime \prime}}=J_{\beta x^{\prime}, \gamma x^{\prime \prime}} .
$$


The result is the antisymmetric tensor,

$$
\Omega_{\alpha x, \beta x^{\prime}}=J_{\alpha x, \beta x^{\prime}}=\left[\begin{array}{cc}
0 & 1 \\
-1 & 0
\end{array}\right] \delta\left(x, x^{\prime}\right) .
$$

Thus e-phase space is also automatically endowed with a symplectic 2-form $\Omega 8$ This is what justifies calling it a phase space in the canonical Hamiltonian sense of the term.

It is worth emphasizing that the complex $J$ and symplectic $\Omega$ structures have been derived and not imposed. The extended metric was derived from premises that are natural in a probabilistic setting (information geometry, spherical symmetry, the topological nature of the field $\Phi$ ) without any reference to principles of mechanics.

Of course, the fact that our premises imply the symplectic and complex structures means that the latter where somehow already implicit in the former. One might therefore consider an alternative approach that inverts the logic: start by assuming the existence of complex and symplectic structures and from these attempt to derive the metric (38) - but this approach has several drawbacks. One is that it would prevent us from gaining any deeper insight into why do symplectic structures arise in physics in the first place. Another is that the metric derived in this way is not unique; the alternative approach leads to a continuum of metrics of which (38) is a member [30. Our premises are more restrictive than merely imposing complex and symplectic structures.

Poisson brackets- The availability of the symplectic 2-form $\Omega_{\alpha x, \beta x^{\prime}}$ leads to the canonical Hamilton-Jacobi formalism. For example, by raising indices one constructs a tensor $\Omega^{\alpha x, \beta x^{\prime}}$ which, as can be easily checked, has the same components as (49). Indeed,

$$
\Omega^{\alpha x, \beta x^{\prime}} \Omega_{\beta x^{\prime}, \gamma x^{\prime \prime}}=\Omega_{\beta x^{\prime}}^{\alpha x} \Omega_{\gamma x^{\prime \prime}}^{\beta x^{\prime}}=-\delta^{\alpha x}{ }_{\gamma x^{\prime \prime}} .
$$

To see its utility consider the gradient 1-form $\tilde{\nabla} F$ of a generic functional $F[\rho, \Phi]$. Its components are

$$
\tilde{\nabla}_{\alpha x} F=\left(\frac{\delta F}{\delta \rho(x)}, \frac{\delta F}{\delta \Phi(x)}\right) .
$$

The action of $\Omega^{\alpha x, \beta x^{\prime}}$ on any two 1-forms $\tilde{\nabla} F_{1}$ and $\tilde{\nabla} F_{2}$ is the Poisson bracket,

$$
\Omega^{\alpha x, \beta x^{\prime}} \tilde{\nabla}_{\alpha x} F_{1} \tilde{\nabla}_{\beta x^{\prime}} F_{2}=\int d x\left(\frac{\delta F_{1}}{\delta \rho(x)} \frac{\delta F_{2}}{\delta \Phi(x)}-\frac{\delta F_{2}}{\delta \rho(x)} \frac{\delta F_{1}}{\delta \Phi(x)}\right)=\left\{F_{1}, F_{2}\right\} .
$$

The special case $F_{1}=\rho$ and $F_{2}=\Phi$,

$$
\left\{\rho(x), \Phi\left(x^{\prime}\right)\right\}=\delta\left(x, x^{\prime}\right), \quad \text { and } \quad\left\{\rho(x), \rho\left(x^{\prime}\right)\right\}=\left\{\Phi(x), \Phi\left(x^{\prime}\right)\right\}=0 ，
$$

shows that $\rho(x)$ and $\Phi(x)$ are canonically conjugate variables.

\footnotetext{
$U$.

${ }^{8}$ It is easy to check that $\Omega$ is also $J$-invariant: $\Omega\left[V_{J}, U_{J}\right]=\Omega[V, U]$ for all vectors $V$ and
} 
Complex coordinates- The availability of a complex structure suggests introducing complex coordinates

$$
\Psi=\rho^{1 / 2} \exp (i \Phi / \hbar) \quad \text { and } \quad \Psi^{*}=\rho^{1 / 2} \exp (-i \Phi / \hbar) .
$$

Indeed, the metric (38) takes a very simple form,

$$
\delta \ell^{2}=2 \hbar \int d x \delta \Psi^{*} \delta \Psi
$$

The corresponding Poisson brackets,

$$
\left\{\Psi(x), \Psi^{*}\left(x^{\prime}\right)\right\}=\frac{1}{i \hbar} \delta\left(x, x^{\prime}\right), \quad \text { and } \quad\left\{\Psi(x), \Psi\left(x^{\prime}\right)\right\}=\left\{\Psi^{*}(x), \Psi^{*}\left(x^{\prime}\right)\right\}=0,
$$

show that $i \hbar \Psi^{*}(x)$ is the momentum that is canonically conjugate to the complex coordinate $\Psi(x)$.

\section{The e-Hamiltonian}

According to entropic dynamics the evolution of $\rho$ is given by the FP eq.(30). Now we seek a criterion to update the phase field $\Phi$ - which amounts to updating the constraints (5) and (6). The joint dynamics of $\rho$ and $\Phi$ must reproduce the FP eq.(30); it is also natural to require that it preserve the metric, complex, and symplectic structures of e-phase space. Such a dynamics is a Hamiltonian flow in e-phase space.

The construction of the ensemble Hamiltonian - or e-Hamiltonian - involves three ingredients. First, if temporal distance has to do with whether we can detect a change from one state to another. The question is: Does a small change make any difference? Or, can we distinguish the new pair $[\rho+\delta \rho, \Phi+\delta \Phi]$ from the old $[\rho, \Phi]$ ? This is precisely the kind of issue that the metric (38) has been designed to address. Then it is only natural that the generator of change be in some way related to the e-phase metric. Second, in ED entropic time is constructed so that time (duration) is defined by a clock that is provided by the system itself. More precisely, time is measured by the fluctuations of free particles. It is only natural to require that the generator of time translations be defined in terms of the same clock. And third, the e-Hamiltonian has to agree with (31) in order to reproduce the ED evolution of $\rho$, eq.(30).

To implement the first condition we note that in ED position plays the privileged role of being the only ontic variable. We will therefore consider a small change of state $\{\delta \rho, \delta \Phi\}$ when the position is slightly shifted by $\delta x^{A}(x)$,

$$
\delta \rho=\partial_{A} \rho \delta x^{A},
$$

The change in $\Phi$ is given by a similar expression except that in order to compare neighboring phases we have to keep track of the gauge connection $A_{A}$. The gauge invariant change in $\Phi$ is

$$
\delta \Phi=\left(\partial_{A} \Phi+\bar{A}_{A}\right) \delta x^{A}
$$


Substituting into (38) gives,

$$
\delta \ell^{2}=\frac{4}{\hbar} \int d x h_{A B} \delta x^{A} \delta x^{B},
$$

where

$$
h_{A B}=\frac{1}{2} \rho\left(\partial_{A} \Phi-\bar{A}_{A}\right)\left(\partial_{B} \Phi-\bar{A}_{B}\right)+\frac{\hbar^{2}}{8 \rho} \partial_{A} \rho \partial_{B} \rho .
$$

Up to the irrelevant factor of $4 / \hbar$, the tensor $h_{A B}$ is the induced metric in configuration space - it measures distinguishability under small displacements.

The second requirement - that duration be measured by fluctuations - is implemented by demanding that the shift $\delta x^{A}$ be associated to fluctuations in a short time interval $\Delta t 9$ Recalling (8) and (24), we see that as $\Delta t \rightarrow 0$, the product $\delta x^{A} \delta x^{B}$ converges in probability to $\left\langle\delta x^{A} \delta x^{B}\right\rangle$,

$$
\delta x^{A} \delta x^{B} \sim\left\langle\delta x^{A} \delta x^{B}\right\rangle+o(\Delta t)=\hbar m^{A B} \Delta t \delta^{a b}+o(\Delta t) .
$$

(See [14], section §5.) Therefore,

$$
\delta \ell^{2} \sim 4 \Delta t \int d x m^{A B} h_{A B}+o(\Delta t) .
$$

To satisfy the third requirement, we compare (31) with (60) and (62). This suggests an e-Hamiltonian,

$$
\tilde{H}_{0}=\int d x m^{A B} h_{A B}
$$

that incorporates all the desired symmetries and even includes the quantum potential term with the right coefficient. However, since the argument relies on the short time evolution - the dynamics of free particles - the construction cannot be expected to reproduce an interaction terms such as a standard scalar potential $V(x)$. Therefore to account for the possibility of additional interactions we introduce an additional potential term $V(x) 10$

$$
\tilde{H}[\rho, \Phi]=\int d x \rho\left[\frac{1}{2} m^{A B}\left(\partial_{A} \Phi-\bar{A}_{A}\right)\left(\partial_{B} \Phi-\bar{A}_{B}\right)+\frac{\hbar^{2}}{8 \rho^{2}} m^{A B} \partial_{A} \rho \partial_{B} \rho+V(x)\right] .
$$

The corresponding Hamilton equations reproduce the FP eq.(30),

$$
\partial_{t} \rho=\frac{\delta \tilde{H}}{\delta \Phi}=-\partial_{A}\left[\rho m^{A B}\left(\partial_{B} \Phi-\bar{A}_{B}\right)\right]
$$

and the analogue of the Hamilton-Jacobi equation,

$$
\partial_{t} \Phi=-\frac{\delta \tilde{H}}{\delta \rho}=-\frac{1}{2} m^{A B}\left(\partial_{A} \Phi-\bar{A}_{A}\right)\left(\partial_{B} \Phi-\bar{A}_{B}\right)+\frac{\hbar^{2}}{2} m^{A B} \frac{\partial_{A} \partial_{B} \rho^{1 / 2}}{\rho^{1 / 2}}-V .
$$

\footnotetext{
${ }^{9} \mathrm{I}$ am grateful to S. Ipek and K. Vanslette for a very useful suggestions on this issue.

${ }^{10} \mathrm{~A}$ scalar potential such as $\rho(x) V(x)$ would arise naturally in a model in which the electromagnetic field is represented by dynamical degrees of freedom.
} 
We can now combine $\rho$ and $\Phi$ into the wave function, $\Psi=\rho^{1 / 2} \exp (i \Phi / \hbar)$. Expressing (65) and (66) in terms of $\Psi$ leads to the Schrödinger equation.

However, there is an equivalent but simpler derivation: use complex coordinates (54) from the start. Use (57) and (58) to find

$$
\delta \Psi=D_{A} \Psi \delta x^{A},
$$

where

$$
D_{A}=\partial_{A}-\frac{i}{\hbar} \bar{A}_{A}=D_{n a}=\frac{\partial}{\partial x_{n}^{a}}-i \beta_{n} A_{a}\left(x_{n}\right) .
$$

Substitute into (55), and use (61) to get,

$$
\delta \ell^{2} \sim 4 \Delta t \int d x \frac{\hbar^{2}}{2} m^{A B}\left(D_{A} \Psi\right)^{*} D_{B} \Psi+o(\Delta t),
$$

which leads to the same e-Hamiltonian as eq.(64),

$$
\tilde{H}\left[\Psi, \Psi^{*}\right]=\int d x \Psi^{*}\left[-\frac{\hbar^{2}}{2} m^{A B} D_{A} D_{B}+V(x)\right] \Psi
$$

The Hamilton equation,

$$
\partial_{t} \Psi(x)=\frac{\delta \tilde{H}}{\delta\left(i \hbar \Psi^{*}(x)\right)},
$$

is the Schrödinger equation,

$$
i \hbar \partial_{t} \Psi=-\frac{\hbar^{2}}{2} m^{A B} D_{A} D_{B} \Psi+V \Psi .
$$

In more standard notation it reads

$$
i \hbar \partial_{t} \Psi=-\sum_{n} \frac{\hbar^{2}}{2 m_{n}} \delta^{a b} D_{n a} D_{n b} \Psi+V \Psi .
$$

At this point we can finally provide the physical interpretation of the various constants introduced along the way: $\hbar$ is Planck's constant, $m_{n}$ are the particles' masses, and the $\beta_{n}$ are related to the particles' electric charges $q_{n}$ by

$$
\beta_{n}=\frac{q_{n}}{\hbar c}
$$

\section{On linearity, single-valuedness, and charge quantization}

Earlier we remarked on the tension between the probabilistic and linear structures in QM. As described by eqs. (65) and (66), ED is a fully probabilistic theory and its representation in terms of the Schrödinger equation (173) is clearly linear. However, this does not yet guarantee the full compatibility of probability with 
linearity. It is clear that if $\Psi_{1}$ and $\Psi_{2}$ are two solutions of eq. (73) then the linear combination

$$
\Psi_{3}=\alpha_{1} \Psi_{1}+\alpha_{2} \Psi_{2}
$$

is also a solution. The problem is that even when $\left|\Psi_{1}\right|^{2}=\rho_{1}$ and $\left|\Psi_{2}\right|^{2}=\rho_{2}$ are probabilities, it is not generally true that $\left|\Psi_{3}\right|^{2}$ will be a probability too.

To see the difficulty note that for generic choices of $\beta_{n}$ the wave functions $\Psi$ are in general multi-valued. As one moves in a closed loop $\Gamma$ in configuration space the wave function changes by a phase factor,

$$
\Psi \rightarrow \Psi^{\prime}=e^{i \delta} \Psi
$$

The superposition of $\Psi_{1}$ and $\Psi_{2}$ is multi-valued too,

$$
\Psi_{3} \rightarrow \Psi_{3}^{\prime}=\alpha_{1} e^{i \delta_{1}} \Psi_{1}+\alpha_{2} e^{i \delta_{2}} \Psi_{2}
$$

The problem is that even if the magnitudes $\left|\Psi_{1}\right|^{2}=\rho_{1}$ and $\left|\Psi_{2}\right|^{2}=\rho_{2}$ are single-valued (because they are probability densities), $\left|\Psi_{3}\right|^{2}$ will in general turn out to be multi-valued. Indeed,

$$
\left|\Psi_{3}\right|^{2}=\left|\alpha_{1}\right|^{2} \rho_{1}+\left|\alpha_{2}\right|^{2} \rho_{2}+2 \operatorname{Re}\left[\alpha_{1} \alpha_{2}^{*} \Psi_{1} \Psi_{2}^{*}\right]
$$

changes into

$$
\left|\Psi_{3}^{\prime}\right|^{2}=\left|\alpha_{1}\right|^{2} \rho_{1}+\left|\alpha_{2}\right|^{2} \rho_{2}+2 \operatorname{Re}\left[\alpha_{1} \alpha_{2}^{*} e^{i\left(\delta_{1}-\delta_{2}\right)} \Psi_{1} \Psi_{2}^{*}\right]
$$

so that in general

$$
\left|\Psi_{3}^{\prime}\right|^{2} \neq\left|\Psi_{3}\right|^{2}
$$

which precludes the interpretation of $\left|\Psi_{3}\right|^{2}$ as a probability. The problem does not arise if the phase $\delta$ in (76) has the same value for all wave functions. Furthermore, since for some wave functions we have $\delta=0$, it must be that $\delta=0$ for all wave functions. Therefore, the condition of compatibility of the probabilistic and linear structures is that the wave functions be single-valued.

The single-valuedness condition is

$$
\Delta \frac{\Phi}{\hbar}=\oint_{\Gamma} d \ell^{A} \partial_{A} \frac{\Phi}{\hbar}=2 \pi \nu(\Gamma)
$$

where $\nu(\Gamma)$ is an integer. To determine the choice of constraints - that is, to determine the values of the $\beta_{n}$ - that lead to single-valuedness consider a closed loop $\Gamma_{n}$ in which all particles but the $n$th are kept fixed. Since $\chi$ is an angle,

$$
\Delta \chi=\oint_{\Gamma_{n}} d \ell_{n}^{a} \partial_{n a} \chi=2 \pi \nu\left(\Gamma_{n}\right)
$$

where $\nu\left(\Gamma_{n}\right)$ is an integer that depends on the loop $\Gamma_{n}$. Furthermore, since $\phi(x)$ and $\log \rho(x)$ are single-valued, from (29), we have

$$
\Delta \frac{\Phi}{\hbar}=\oint_{\Gamma_{n}} d \ell_{n}^{a} \partial_{n a} \frac{\Phi}{\hbar}=\beta_{n} \oint_{\Gamma_{n}} d \ell_{n}^{a} \partial_{n a} \chi=2 \pi \nu\left(\Gamma_{n}\right) \beta_{n} .
$$


Comparing with eq. (81) for any arbitrary loops shows that $\beta_{n}$ must be an integer. Therefore, the choice of $\kappa_{n}$ in the gauge constraints (6) that leads to compatibility of probabilistic and linear structures is that the corresponding Lagrange multipliers $\beta_{n}$ be integers.

Quantized electric charges Equation (74) then shows that electric charges must occur in integer multiples of a basic charge $q=\hbar c 11$

$$
q_{n}=\beta_{n} \hbar c .
$$

Therefore, the condition for compatibility of the probabilistic and linear structures that leads to full equivalence between ED and the QM of charged particles is that charges be quantized.

\section{Conclusions}

On quantum geometry- The information geometry of the space of probabilities can be naturally extended to the full e-phase space of probabilities and phases. This reproduces the Riemannian, complex and symplectic structures characteristic of QM. The ED that preserves these structures while recognizing the privileged ontic role of position is the Schrödinger equation.

On charge quantization and single-valuedness- There is a deep connection between the probabilistic structure of QM, its linearity, the singlevaluedness of wave functions, and the quantization of charge. Full equivalence between ED and QM is achieved when we adopt constraints that reflect the intimate relation between quantum phases and gauge invariance, and also reflect the empirical fact that charges are quantized. If, alternatively, we take linearity as the "empirical" input, then we deduce charge quantization.

Acknowledgments I would like to thank M. Abedi, D. Bartolomeo, C. Cafaro, N. Carrara, N. Caticha, S. DiFranzo, A. Giffin, S. Ipek, D.T. Johnson, K. Knuth, S. Nawaz, M. Reginatto, C. Rodríguez, and K. Vanslette, for many valuable discussions.

\section{References}

[1] H.P. Stapp, Am. J. Phys. 40, 1098 (1972).

[2] M. Schlösshauer, Rev. Mod. Phys. 76, 1267 (2004).

[3] G. Jaeger, Entanglement, Information, and the Interpretation of Quantum Mechanics (Springer-Verlag, Berlin Heidelberg 2009).

\footnotetext{
${ }^{11}$ We can change to more conventional units by rescaling charges and potentials according to $\lambda q_{n}=q_{n}^{\prime}$ and $A_{a} / \lambda=A_{a}^{\prime}$ so that $q_{n} A_{a}=q_{n}^{\prime} A_{a}^{\prime}$. For conventional units such that the basic charge is $q^{\prime}=e / 3$ with $\alpha=e^{2} / \hbar c=1 / 137$ the scaling factor is $\lambda=\frac{1}{3}(\alpha / \hbar c)^{1 / 2}$.
} 
[4] M. S. Leifer, Quanta 3, 67 (2014); arXiv.org: 1409.1570.

[5] E. T. Jaynes, Phys. Rev. 106, 620 and 108, 171 (1957);

[6] E. T. Jaynes: Papers on Probability, Statistics and Statistical Physics, Ed. by R. D. Rosenkrantz (Reidel, Dordrecht, 1983).

[7] E. T. Jaynes, Probability Theory: The Logic of Science edited by G. L. Bretthorst (Cambridge UP, 2003).

[8] A. Caticha, Entropic Inference and the Foundations of Physics (EBEB 2012, São Paulo, Brazil); http://www.albany.edu/physics/ACaticha-EIFP-book.pdf.

[9] A. Caticha, J. Phys. A: Math. Theor. 44, 225303 (2011); arXiv.org:1005.2357.

[10] A. Caticha, J. Phys.: Conf. Ser. 504, 012009 (2014); arXiv.org:1403.3822.

[11] A. Caticha, D. Bartolomeo, M. Reginatto, AIP Conf. Proc. 1641, 155 (2015); arXiv.org:1412.5629.

[12] A. Caticha, Entropy 17, 6110 (2015); arXiv.org:1509.03222.

[13] N. Carrara, A. Caticha, "Quantum phases in entropic dynamics," arXiv.org:1708.08977.

[14] E. Nelson, Quantum Fluctuations (Princeton UP, Princeton, 1985).

[15] S. Adler, Quantum Theory as an Emergent Phenomenon (Cambridge UP, Cambridge, 2004).

[16] L. Smolin, "Could quantum mechanics be an approximation to another theory?" arXiv.org/abs/quant-ph/0609109.

[17] L. de la Peña, A.M. Cetto, The Emerging Quantum: The Physics Behind Quantum Mechanics (Springer, 2014).

[18] G. Grössing, Phys. Lett. A, 372, 4556 (2008), arXiv:0711.4954; G. Grössing et al, J. Phys. Conf. Ser. 361, 012008 (2012).

[19] See e.g., the proceedings of the conference on EmQm15: Emergent Quantum Mechanics 2015, J. Phy. Conf. Ser. 701 (2016) http://iopscience.iop.org/issue/1742-6596/701/1.

[20] G. 't Hooft, The Cellular Automaton Interpretation of Quantum Mechanics (Springer, 2016).

[21] W. K. Wootters, Phys. Rev. D 23, 357-362 (1981).

[22] A. Caticha, Phys. Lett. A 244, 13-17 (1998); Phys. Rev. A 57, 1572-1582 (1998); Found. Phys. 30, 227-251 (2000). 
[23] C. Brukner, A. Zeilinger, "Information and Fundamental Elements of the Structure of Quantum Theory" in Time, Quantum, Information, ed. L. Castell and O. Ischebeck, (Springer 2003); arXiv:quant-ph/0212084.

[24] M. Mehrafarin, Int. J. Theor. Phys., 44, 429 (2005); arXiv:quant-ph/0402153.

[25] R. Spekkens, Phys. Rev. A 75, 032110 (2007).

[26] P. Goyal, New J. Phys., 12, 023012 (2010).

[27] P. Goyal, K. Knuth, and J. Skilling, Phys. Rev. A 81, 022109 (2010).

[28] L. Hardy, "Reconstructing Quantum Theory," in Quantum Theory: Informational Foundations and Foils ed. G. Chiribella and R. Spekkens (Springer, 2015); arXiv:1303.1538.

[29] M. Reginatto and M.J.W. Hall, AIP Conf. Proc. 1443, 96 (2012); arXiv:1108.5601

[30] M. Reginatto and M.J.W. Hall, AIP Conf. Proc. 1553, 246 (2013); arXiv:1207.6718

[31] T. Takabayasi, Prog. Theor. Phys. 8, 143 (1952).

[32] T. C. Wallstrom, Found. Phys. Lett. 2, 113 (1989).

[33] T. C. Wallstrom, Phys. Rev. A 49, 1613 (1994).

[34] T. Takabayasi, Prog. Theor. Phys. 70, 1-17 (1983).

[35] W. Pauli, Helv. Phys. Acta 12, 147 (1939) and W. Pauli, General Principles of Quantum Mechanics (Springer-Verlag, Berlin 1980).

[36] E. Merzbacher, Am. J. Phys. 30, 237 (1962).

[37] E. Nelson, Lect. Notes Phys. 100, 168 (Springer-Verlag, Berlin, 1979).

[38] T. W. B. Kibble, Comm. Math. Phys. 65, 189-201 (1979).

[39] A. Heslot, Phys. Rev. D31, 1341-1348 (1985).

[40] J. Anandan and Y. Aharonov, Phys. Rev. Lett. 65, 1697-1700 (1990).

[41] R. Cirelli, A. Manià, and L. Pizzochero, J. Math. Phys. 31, 2891-2897 (1990); J. Math. Phys. 31, 2898-2903 (1990).

[42] S. Abe, Phys. Rev. A 46, 1667 (1992).

[43] A. Ashtekar and T. A. Schilling, "Geometrical Formulation of Quantum Mechanics," in On Einstein's Path, ed. by A. Harvey (Springer, New York, 1998). 
[44] D. J. Brodie and L. P. Hughston, Phil. Trans. R. Soc. London A 454, 2445 (1998); arXiv:gr-qc/9701051.

[45] D.T. Johnson and A. Caticha, AIP Conf. Proc. 1443, 104 (2012); arXiv:1108.2550

[46] K. Vanslette and A. Caticha, AIP Conf. Proc. 1853, 090003 (2017); arXiv:1701.00781.

[47] S. Nawaz and A. Caticha, AIP Conf. Proc. 1443, 112 (2012); arXiv:1108.2629

[48] D. Bartolomeo and A. Caticha, AIP Conf. Proc. 1757, 030002 (2016); arXiv.org:1512.09084.

[49] D. Bartolomeo and A. Caticha, J. Phys: Conf. Series 701, 012009 (2016); arXiv.org:1603.08469.

[50] A. Demme and A. Caticha, AIP Conf. Proc. 1853, 090001 (2017); arXiv.org:1612.01905.

[51] S. Nawaz, M. Abedi, and A. Caticha, AIP Conf. Proc. 1757, 030004 (2016); arXiv.org:1601.01708.

[52] S. Ipek and A. Caticha, AIP Conf. Proc. 1641, 345 (2015); arXiv.org:1412.5637.

[53] S. Ipek, M. Abedi, and A. Caticha, AIP Conf. Proc. 1853, 090002 (2017).

[54] J. Barbour, Class. Quant. Grav. 11, 2853 and 2875 (1994); "The emergence of time and its arrow from timelessness" in Physical Origins of Time Asymmetry, eds. J. Halliwell et al, (Cambridge University Press, Cambridge 1994).

[55] Price, H. Time's Arrow and Archimedes' Point, Oxford UP, New York 1996.

[56] Zeh, H.D. The Physical Basis of the Direction of Time, Springer, 2002.

[57] S. Amari, Differential-Geometrical Methods in Statistics (Springer-Verlag, 1985).

[58] C. Lanczos, The Variational Principles of Mechanics (4th edition, Dover, New York 1986).

[59] L. L. Campbell, Proc. Am. Math. Soc. 98, 135 (1986). 